\title{
Vibration control via stiffness switching of magnetostrictive transducers
}

\author{
Justin J. Scheidler $^{a}$, Vivake M. Asnani $^{b}$, Marcelo J. Dapino ${ }^{c}$ \\ ${ }^{a}$ Universities Space Research Association, NASA Glenn Research Center, Cleveland, OH \\ 44135 \\ ${ }^{b}$ Rotating and Drive Systems Branch, Materials and Structures Division, NASA Glenn \\ Research Center, Cleveland, $\mathrm{OH} 44135$ \\ ${ }^{c}$ NSF I/UCRC on Smart Vehicle Concepts, Department of Mechanical and Aerospace \\ Engineering, The Ohio State University, Columbus, OH, USA, 43210
}

\begin{abstract}
In this paper, a computational study is presented of structural vibration control that is realized by switching a magnetostrictive transducer between high and low stiffness states. Switching is accomplished by either changing the applied magnetic field with a voltage excitation or changing the shunt impedance on the transducer's coil (i.e., the magnetostrictive material's magnetic boundary condition). Switched-stiffness vibration control is simulated using a lumped mass supported by a damper and the magnetostrictive transducer (mount), which is represented by a nonlinear, electromechanical model. Free vibration of the mass is calculated while varying the mount's stiffness according to a reference switched-stiffness vibration control law. The results reveal that switching the magnetic field produces the desired change in stiffness, but also an undesired actuation force that can significantly degrade the vibration control. Hence, a modified switched-stiffness control law that accounts for the actuation force is proposed and implemented for voltage-controlled stiffness switching. The influence of the magnetomechanical bias condition is also discussed. Voltage-controlled stiffness switching is found to introduce damping equivalent to a viscous damping factor up to about 0.13 ; this is shown to primarily result from active vibration reduction caused by the actuation force. The merit of magnetostrictive switched-stiffness vibration control is then quantified by comparing the results of voltageand shunt-controlled stiffness switching to the performance of optimal magnetostrictive shunt damping. For the cases considered, optimal resistive shunt damping performed considerably better than both voltage- and shunt-controlled stiffness switching.
\end{abstract}

Keywords: magnetostrictive materials, switched-stiffness vibration control, stiffness tuning, shunt damping, Terfenol-D

\section{INTRODUCTION}

Magnetostrictive materials, such as Galfenol $\left(\mathrm{Fe}_{1-x} \mathrm{Ga}_{x}, 0.13 \leq x \leq 0.29\right)$ and Terfenol-D $\left(\mathrm{Tb}_{x} \mathrm{Dy}_{1-x} \mathrm{Fe}_{y}\right.$, $x \approx 0.3, y \approx 2$ ), exhibit a bi-directional coupling between magnetic and mechanical domains, thereby providing actuation and sensing effects. These materials also exhibit stress- and magnetic field-dependent elastic moduli, which result from the superposition of magnetoelastic strain and purely elastic strain. ${ }^{1}$ The moduli can be controlled precisely and remotely using electromagnets. This effect can be utilized for many vibration control applications, such as adaptive suspensions, variable-frequency mechanical oscillators, ${ }^{2}$ semi-active vibration absorbers, ${ }^{3-6}$ and switched-stiffness vibration control. ${ }^{7,8}$ Recently, Scheidler et al. ${ }^{9}$ developed a magnetostrictive transducer having a stiffness that can be controlled in real-time to enable continuous stiffness tracking or stiffness switching.

justin.j.scheidler@nasa.gov; phone: 1-216-433-6440; fax: 1-216-433-3954 
In this paper, the usefulness of a magnetostrictive transducer with dynamically-tunable stiffness is studied by applying the transducer to switched-stiffness vibration control of a single-degree-of-freedom mechanical system. Switched-stiffness vibration control is a simple form of stiffness control in which vibration is attenuated via coordinated switching between high and low stiffness states. ${ }^{7,8}$ Two distinct methods of stiffness switching are considered: changing the bias magnetic field using a voltage excitation and changing the impedance of an electrical shunt connected to the transducer's electromagnet. A computational study is presented to investigate the uncontrolled and controlled free vibration responses of the mass. First, a model is introduced that quantifies the nonlinear electromechanical behavior of the magnetostrictive transducer and its effect on the vibration of the mechanical system. Constitutive nonlinearities are incorporated by using a fully-nonlinear constitutive model for magnetostrictive materials from the literature. Then, a discussion of the control algorithm is presented and modifications to the algorithm are proposed to account for the magnetostrictive force generated by the transducer. Next, control of the mass's free vibration using voltagecontrolled stiffness switching is calculated and the effect of the magnetostrictive force is discussed. The effectiveness of voltage-controlled stiffness switching is then compared to that of shunt-controlled stiffness switching and optimal resistive shunt damping.

\section{MODEL DEVELOPMENT}

Switched-stiffness vibration control using a magnetostrictive transducer is investigated for the mechanical system shown in Fig. 1, where the transducer is used as a spring mount to control the motion $x$ of mass $m$ that is caused by an applied force $F$. Two methods of controlling the transducer's stiffness are considered: (1) an excitation voltage $V$ that produces current $i$, which generates an axial magnetic field $H$ in the magnetostrictive material and (2) an electrical shunt $Z_{\text {sh }}$ with controllable impedance that changes the electrical boundary condition on the coil and thus the magnetic boundary condition imposed on the magnetostrictive material. Due to magnetostriction, the field generated in case (1) also produces an unwanted magnetostrictive force $F_{\text {mag }}$ that excites the mass; the presence of $F_{\text {mag }}$ complicates the vibration control strategy, as discussed in Section 3. A viscous damper $c$ is included to provide conventional mechanical dissipation. First, the equations governing the dynamic, nonlinear, electromechanical response of the system shown in Fig. 1 are derived for the case when both the excitation voltage and shunt are simultaneously connected to the transducer. Then, the equations are simplified for the two aforementioned cases.

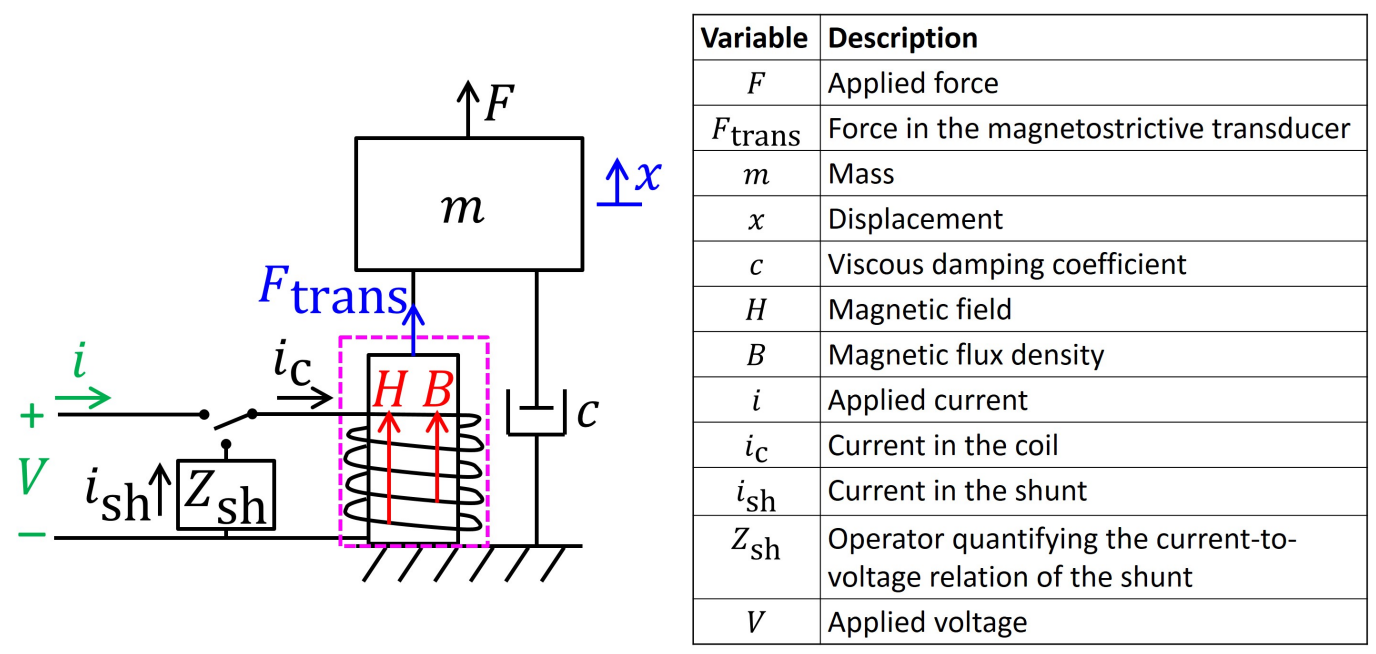

Figure 1: Mechanical system used to investigate switched-stiffness vibration control; the magnetostrictive transducer (dotted magenta box) has electrical inputs (green), magnetic states (red), and mechanical outputs (blue). 
The equation of motion for the mechanical system is

$$
m \frac{d^{2}}{d t^{2}}(\Delta x)+c \frac{d}{d t}(\Delta x)+\Delta F_{\text {trans }}(H, T)=\Delta F,
$$

where $\Delta x$ is the incremental displacement of the mass and $\Delta F_{\text {trans }}$ is the total force imposed by the transducer, which depends on the total field and stress inside the magnetostrictive material,

$$
\begin{aligned}
& T=\Delta T+T_{\text {bias }}, \\
& H=\Delta H+H_{\text {bias }} .
\end{aligned}
$$

Here, $H_{\text {bias }}$ and $T_{\text {bias }}$ are the bias field and stress, respectively. The equation of motion is formulated in incremental form to be consistent with the piezomagnetic equations that govern the transducer's magnetomechanical behavior,

$$
\begin{aligned}
& \Delta B=\mu^{S}(H, T) \Delta H+d(H, T) E^{H}(H, T) \Delta S, \\
& \Delta T=-d(H, T) E^{H}(H, T) \Delta H+E^{H}(H, T) \Delta S,
\end{aligned}
$$

where $\Delta B, \Delta T$, and $\Delta S$ respectively denote the incremental magnetic flux density, stress, and strain along the axis of the magnetostrictive rod, which is described by its magnetic permeability at constant strain $\mu^{S}$, Young's modulus at constant field $E^{H}$, and piezomagnetic coefficient $d$. The permeability at constant strain is related to the permeability at constant stress $\mu^{T}$ through $\mu^{S}=\mu^{T}\left(1-\kappa^{2}\right)$, where $\kappa$ is the magnetomechanical coupling factor. Eqs. (1), (4), and (5) are nonlinear, because the dependence of the material properties on $H$ and $T$ is explicitly considered through the use of a fully-nonlinear constitutive model for magnetostrictive materials. ${ }^{10-12}$ Hereafter, the dependence of the material properties on $H$ and $T$ is dropped from the notation for clarity.

If the magnetic flux leakage and the magnetic reluctance of the transducer's flux return path are assumed to be negligible, the magnetic field in the magnetostrictive rod is $\Delta H=N \Delta i_{\mathrm{c}} / l_{\mathrm{c}}$, where $N$ and $l_{\mathrm{c}}$ are the number of windings in and axial length of the electromagnet, respectively. When the mass vibrates at frequencies sufficiently below the transducer's 1st resonant frequency, $\Delta T$ and $\Delta S$ are uniform and can be calculated as $\Delta F_{\text {trans }} / A_{\mathrm{r}}$ and $\Delta x / l_{\mathrm{r}}$, respectively, where $A_{\mathrm{r}}$ and $l_{\mathrm{r}}$ are the cross-sectional area and length of the magnetostrictive rod, respectively. Insertion of these expressions into Eq. (5) gives

$$
\Delta F_{\text {trans }}=\frac{A_{\mathrm{r}} E^{H}}{l_{\mathrm{r}}} \Delta x-\frac{d N A_{\mathrm{r}} E^{H}}{l_{\mathrm{c}}} \Delta i_{\mathrm{c}}=k^{H} \Delta x-\Theta \Delta i_{\mathrm{c}},
$$

where $k^{H}$ is the transducer's stiffness and $\Theta$ is an electromechanical coupling coefficient. From Eq. (6), the magnetostrictive force can be identified as

$$
\Delta F_{\text {mag }}=-\Theta \Delta i_{\mathrm{c}} .
$$

The Faraday-Lenz law governs the electromotive force $\Delta V_{\text {emf }}$ induced in the transducer's electromagnet due to the magnetic response of the magnetostrictive material,

$$
\Delta V_{\mathrm{emf}}=-N A_{\mathrm{c}} \frac{d}{d t}(\Delta B),
$$

where $A_{\mathrm{c}}$ is the cross-sectional area of the electromagnet. Insertion of Eq. (4) and the expressions for $\Delta S$ and $\Delta H$ into Eq. (8) gives

$$
\Delta V_{\mathrm{emf}}=-\frac{d}{d t}\left(\frac{d N A_{\mathrm{c}} E^{H}}{l_{\mathrm{r}}} \Delta x+\frac{N^{2} A_{\mathrm{c}} \mu^{S}}{l_{\mathrm{c}}} \Delta i_{\mathrm{c}}\right)=-\frac{d}{d t}\left(\Theta \Delta x+L_{\mathrm{c}}^{S} \Delta i_{\mathrm{c}}\right),
$$

where $L_{\mathrm{c}}^{S}$ is the inductance of the transducer at constant strain* and it was assumed that $l_{\mathrm{c}}=l_{\mathrm{r}}$ and $A_{\mathrm{c}}=A_{\mathrm{r}} . L_{\mathrm{c}}^{S}$ is related to the inductance at constant stress $L_{\mathrm{c}}^{T}$ through $L_{\mathrm{c}}^{S}=L_{\mathrm{c}}^{T}\left(1-\kappa^{2}\right)$.

\footnotetext{
${ }^{*}$ This quantity has also been referred to as the self inductance of a magnetostrictive transducer. ${ }^{13}$
} 


\subsection{Stiffness switching via controlled voltages}

When the electrical switch in Fig. 1 is connected to the excitation voltage, the electrical system is characterized by the following equations,

$$
\begin{array}{r}
\Delta i=\Delta i_{\mathrm{c}}, \\
\Delta V-R_{\mathrm{c}} \Delta i_{\mathrm{c}}+\Delta V_{\mathrm{emf}}=0,
\end{array}
$$

where $\Delta i_{\mathrm{c}}$ are $R_{\mathrm{c}}$ are the incremental current in and resistance of the electromagnet, respectively.

The equations governing the electrical and mechanical responses of the voltage-controlled transducer are found by combining Eqs. (1), (6), (9), (10), and (11), resulting in

$$
\begin{aligned}
m \frac{d^{2}}{d t^{2}}(\Delta x)+c \frac{d}{d t}(\Delta x)+k^{H} \Delta x-\Theta \Delta i & =\Delta F, \\
\frac{d}{d t}(\Theta \Delta x)+\frac{d}{d t}\left(L_{\mathrm{c}}^{S} \Delta i\right)+R_{\mathrm{c}} \Delta i & =\Delta V .
\end{aligned}
$$

Eqs. (12) and (13) constitute a nonlinear model that incorporates the mechanical vibration of the mass, the electrical dynamics associated with voltage control of the transducer, the magnetostrictive force, and constitutive nonlinearities.

\subsection{Stiffness switching and shunt damping via electrical shunting}

When the electrical switch in Fig. 1 is connected to the shunt $Z_{\mathrm{sh}}$, the electrical system is characterized by the following equations,

$$
\begin{aligned}
\Delta i_{\mathrm{sh}} & =\Delta i_{\mathrm{c}}, \\
\Delta V_{\mathrm{emf}}-R_{\mathrm{c}} \Delta i_{\mathrm{c}}-Z_{\mathrm{sh}}\left\{\Delta i_{\mathrm{sh}}\right\} & =0,
\end{aligned}
$$

where $\Delta i_{\mathrm{sh}}$ is the incremental current in the shunt and $Z_{\mathrm{sh}}\{\cdot\}$ is an operator that describes the current-tovoltage relation of the shunt; for example, if the shunt is a resistor $R_{\mathrm{sh}}$ and inductor $L_{\mathrm{sh}}$ in series, then

$$
Z_{\mathrm{sh}}\{\cdot\}=\frac{d}{d t}\left(L_{\mathrm{sh}}(\cdot)\right)+R_{\mathrm{sh}}(\cdot)
$$

The equations governing the electrical and mechanical responses of the electrically shunted transducer are found by combining Eqs. (1), (6), (9), (14), and (15),

$$
\begin{array}{r}
m \frac{d^{2}}{d t^{2}}(\Delta x)+c \frac{d}{d t}(\Delta x)+k^{H} \Delta x-\Theta \Delta i=\Delta F, \\
\frac{d}{d t}(\Theta \Delta x)+\frac{d}{d t}\left(L_{\mathrm{c}}^{S} \Delta i_{\mathrm{c}}\right)+R_{\mathrm{c}} \Delta i_{\mathrm{c}}+Z_{\mathrm{sh}}\left\{\Delta i_{\mathrm{sh}}\right\}=0 .
\end{array}
$$

Eqs. (17) and (18) constitute a nonlinear model that incorporates the mechanical vibration of the mass, the electrical dynamics associated with electrical shunting of the transducer, the magnetostrictive force, and constitutive nonlinearities.

As discussed in Section 3.3, shunt-controlled stiffness switching is implemented in this paper using resistive shunts. Thus, the shunt operator is $Z_{\mathrm{sh}}\{\cdot\}=R_{\mathrm{sh}}(\cdot)$ here. After linearizing Eqs. (17) and (18), it can be shown that magnetostrictive shunt damping is analogous to piezoelectric shunt damping. Consequently, the optimal shunt resistance $R_{\mathrm{sh} \text {,opt }}$ is equivalent to that for piezoelectric shunt damping ${ }^{14}$ with the inductive impedance of the transducer being analogous to the capacitive impedance of the piezoelectric,

$$
R_{\mathrm{sh}, \mathrm{opt}}=L_{\mathrm{c}}^{T} \omega_{n}^{H} \sqrt{1-\kappa^{2}}-R_{\mathrm{c}},
$$

where $\omega_{n}^{H}$ is the mechanical natural frequency of the system,

$$
\omega_{n}^{H}=\sqrt{\frac{k^{H}}{m}} .
$$




\subsection{Eddy current effects}

Since the proposed models are nonlinear, the incorporation of mechanically-induced ${ }^{15}$ or field-induced eddy current effects into the models would require that the nonlinear PDE governing magnetic diffusion ${ }^{16}$ be solved concurrently with either Eqs. (12) and (13) (for voltage control) or Eqs. (17) and (18) (for shunting). These effects are not considered here, because this would add a significant amount of complexity to the model. ${ }^{\dagger}$ Hence, the proposed model is accurate when the mass vibrates at a frequency that is below the transducer's mechanically- and field-induced magnetic diffusion cut-off frequencies. Terfenol-D and Galfenol are the magnetostrictive materials that are typically used in uniaxial magnetostrictive transducers. A Terfenol-D rod has magnetic diffusion cut-off frequencies about 40 times greater than those of an equally-sized Galfenol rod. ${ }^{9,15}$ Thus, this paper considers a Terfenol-D-based transducer to improve the accuracy of this assumption.

\section{RESULTS AND DISCUSSION}

This section first presents a modified control law for switched-stiffness vibration control of voltage-controlled magnetostrictive transducers. Then, the control law is implemented to explore the effectiveness and limitations of voltage-controlled stiffness switching. Next, the performance of voltage-controlled stiffness switching is compared to that of shunt-controlled stiffness switching and optimal resistive shunt damping.

Eqs. (12) and (13) are simultaneously solved using a commercial differential equation solver after approximating the time derivatives of $\Theta$ and $L^{S}$ using the 2nd order, backward finite difference method. The solution procedure is iterative and piece-wise linear (i.e., the material properties are updated at the end of each time step). Accordingly, a very small time span is used for each call of the differential equation solver to maintain accuracy.

\subsection{Switched-stiffness vibration control law}

The control law for switched-stiffness vibration control is simple: when the mass is moving toward static equilibrium, switch the stiffness to the soft state; otherwise, switch to the stiff state. ${ }^{7,8}$ In terms of energy, if the stiffness is instantaneously decreased by $\Delta k$ at the $i^{\text {th }}$ displacement maximum $x_{\max , \mathrm{i}}$, the potential energy in the system decreases by $0.5 \Delta k x_{\max , \mathrm{i}}^{2}$. Then, if the stiffness is instantaneously increased by $\Delta k$ at static equilibrium, the potential energy does not increase. Thus, energy in the amount of $0.5 \Delta k x_{\max , \mathrm{i}}^{2}$ is dissipated every half cycle.

In the absence of a magnetostrictive force, the performance of this control method tends to improve as the amplitude of stiffness switching $(\Delta k)$ increases and the time required to switch the stiffness decreases. However, $F_{\text {mag }}$ can have a significant magnitude; thus, it complicates the control method and can produce unexpected motion of the mass. In particular, resonance of the mass can be induced by the control during damped, free vibration, as shown in Fig. 2a. In this case, $F_{\text {mag }}$ overcomes the existing damping and the energy lost due to stiffness switching. This can result when $F_{\text {mag }}$ acts in only one direction (as seen in Fig. $2 \mathrm{~b}$ ), which occurs when the transducer is operated about a variety of bias conditions. Hence, the control law for switched-stiffness vibration control should be modified to that illustrated in Fig. 3 when voltage (or current) control of a magnetostrictive material is used to realize the stiffness changes. Ideally, $F_{\text {mag }}$ is as small as possible and always pushes or pulls the mass toward the static equilibrium position. However, it does not seem possible to satisfy the stiffness and force criteria for every motion condition. As such, for half of the vibration cycle, the field is set to the bias value and stiffness switching is inactive; during this time, $F_{\operatorname{mag}}$ is approximately zero.

To determine a magnetomechanical bias condition for which the tuning conditions shown in Fig. 3 will be satisfied, it is beneficial to reference the Young's modulus of the magnetostrictive material and the electromechanical coupling coefficient of the transducer, which are depicted in Fig. 4. For a given magnetic field change in Terfenol-D, a larger change in Young's modulus is obtained at a moderate to high bias

\footnotetext{
${ }^{\dagger}$ If the proposed models are linearized, eddy current effects can be easily considered by replacing the magnetomechanical material properties with effective properties that are complex-valued and frequency dependent. ${ }^{15,17}$
} 


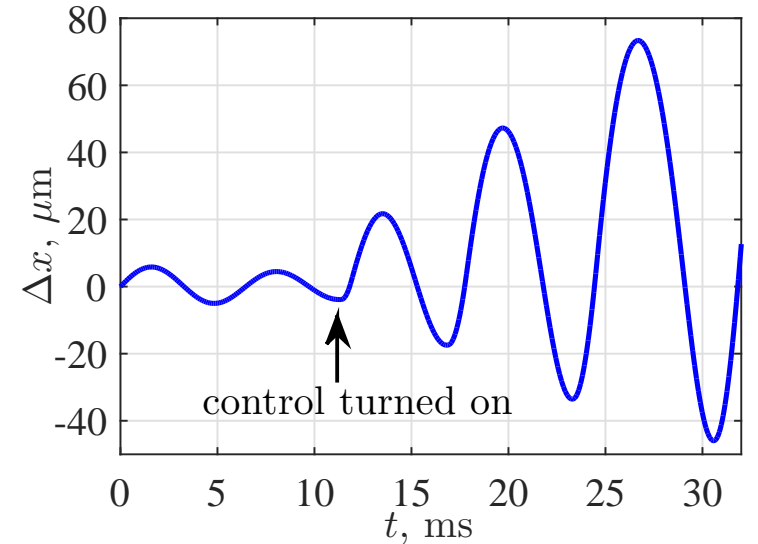

(a)

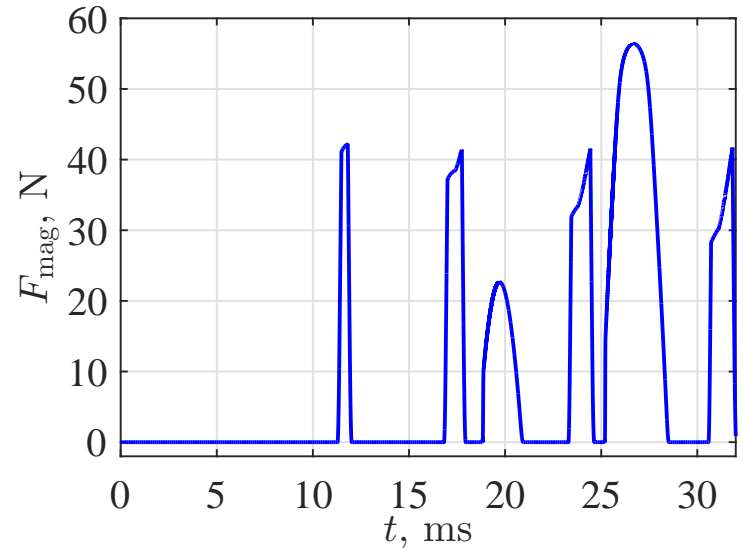

(b)

Figure 2: Mechanical resonance induced by switched-stiffness vibration control due to the unwanted magnetostrictive force; model parameters given in Table $1 ; H=0_{-0}^{+35} \mathrm{kA} / \mathrm{m}$.

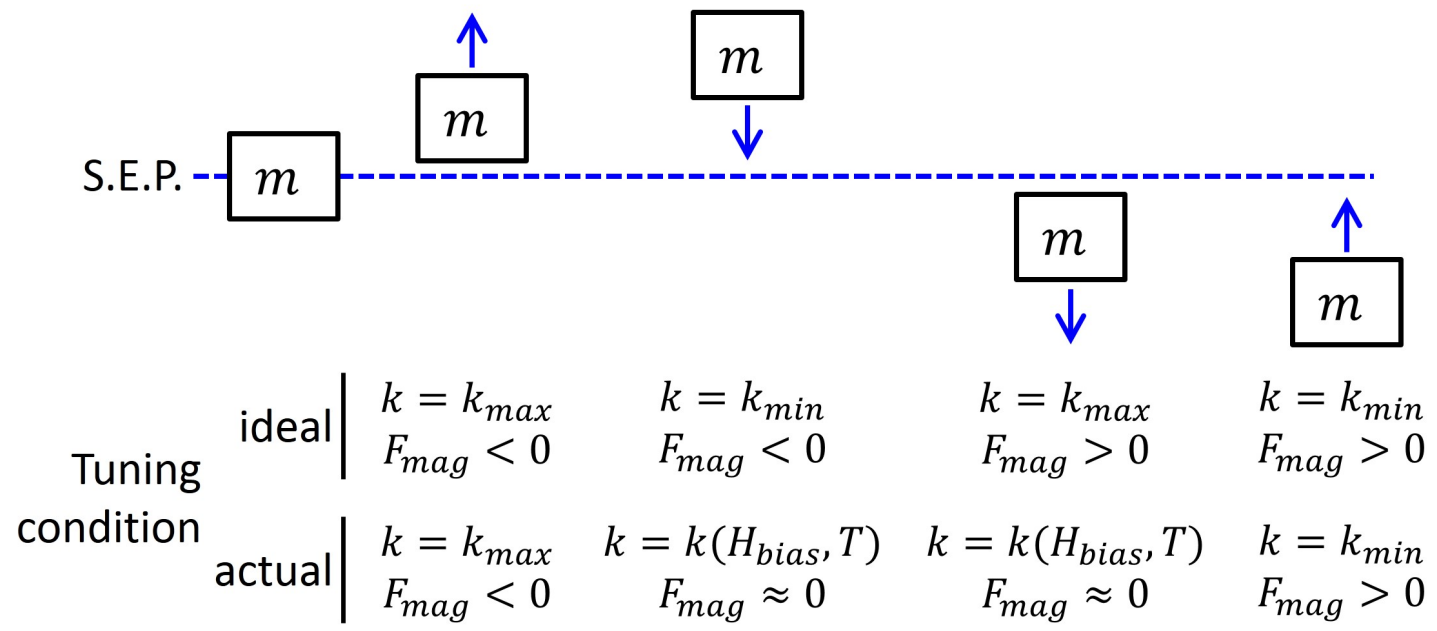

Figure 3: Tuning conditions of the magnetostrictive transducer needed to realize switched-stiffness vibration control in the presence of a magnetostrictive force; S.E.P. denotes the static equilibrium position.

compression than at a small compression. Larger bias compressions also help to prevent unloading of the brittle Terfenol-D rod during dynamic operation. Thus, a moderate to high bias compressive stress is considered herein. Fig. 4b shows that the electromechanical coupling coefficient is always positive for positive magnetic fields. ${ }^{\ddagger}$ According to Eq. $(7), F_{\text {mag }}$ can therefore only change sign if $\Delta i$ changes polarity during stiffness switching; this implies that the bias magnetic field should be between the maximum and minimum tuning fields. At moderate to high bias compressive stress, $\Theta$ decreases rapidly with field at low bias fields; this suggests that the bias field should be closer to the maximum tuning field $H_{\max }$ than to the minimum tuning field $H_{\mathrm{min}}$, in order for $F_{\mathrm{mag}}$ to have comparable positive and negative peak values. Therefore, the bias and tuning fields are reported as

$$
\left(H_{\text {bias }}\right)_{H_{\min }-H_{\text {bias }}}^{H_{\max }-H_{\text {bias }}} .
$$

For example, $H=30_{-15}^{+25} \mathrm{kA} / \mathrm{m}$ means that the voltage is controlled such that the steady-state field is switched from a bias of $30 \mathrm{kA} / \mathrm{m}$ down to $15 \mathrm{kA} / \mathrm{m}$ and up to $55 \mathrm{kA} / \mathrm{m}$.

\footnotetext{
${ }^{\ddagger}$ For negative magnetic fields (not shown in Fig. 4b), the electromechanical coupling coefficient is always negative.
} 


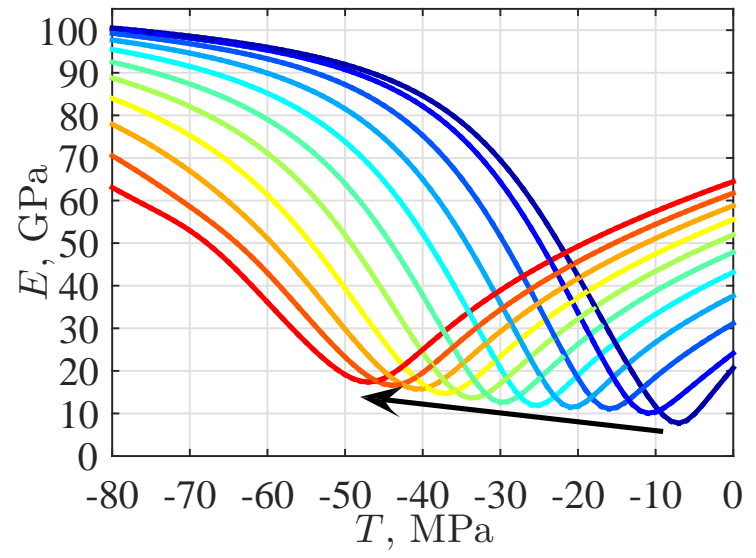

(a)

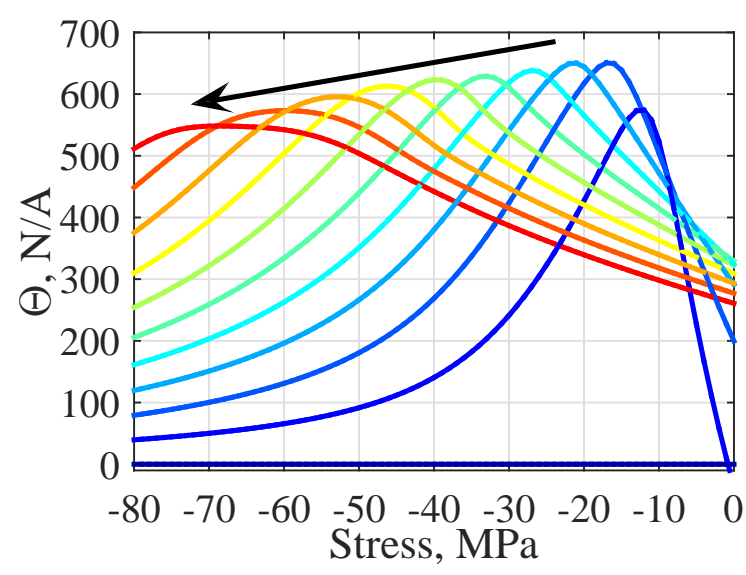

(b)

Figure 4: Stress and magnetic field dependence of the (a) Young's modulus of a Terfenol-D rod and (b) electromechanical coupling coefficient of a Terfenol-D-based transducer calculated using the DEA model in; ${ }^{10}$ from blue to red (the direction of the arrow), $H$ increases from 0 to $100 \mathrm{kA} / \mathrm{m}$ in increments of $10 \mathrm{kA} / \mathrm{m} ; A_{\mathrm{c}}=1.27 \times 10^{-4} \mathrm{~m}^{2}, l_{\mathrm{r}}=0.144 \mathrm{~m}$, and $N=1840$ turns (the minimum number needed to generate $85 \mathrm{kA} / \mathrm{m}$ at steady state with 22 AWG wire).

\subsection{Voltage-controlled stiffness switching}

The modified control method was applied to the undamped, free vibration of the mass. The results are shown in Fig. 5, where an uncontrolled response is compared to two controlled responses. The model parameters common to all three cases are given in Table 1. In both of the controlled responses, switched-stiffness vibration control introduces a significant amount of damping. Although the controlled responses do not decay according to a simple, viscously damped system, the control-induced damping can be approximately described using an effective viscous damping factor calculated by logarithmic decrement; the results are shown in Table 2. On close inspection, controlled response 1 initially decays faster than controlled response 2 , but it converges to a steady oscillation having a small amplitude. This is caused by a small imbalance between the positive and negative peak values of the $F_{\text {mag }}$, as seen in Fig. 5d. If another damping mechanism is present (e.g., shunt damping or $c \neq 0$ ), this oscillation can be easily eliminated by turning off the control when the steady oscillation is reached. Fig. 5b illustrates that under voltage control, the Young's modulus, and thus the stiffness, can vary considerably while operating at a given voltage state. This is due, in part, to the variation in magnetic field (Fig. 5c) that results from a changing current in the coil. This suggests that the vibration control performance should improve if the applied current is controlled rather than the applied voltage.

Table 1: Model parameters for switched-stiffness vibration control modeling.

\begin{tabular}{cccccccc}
\hline$d t, \mu \mathrm{s}$ & $m, \mathrm{~kg}$ & $c, \mathrm{Ns} / \mathrm{m}$ & $R_{\mathrm{c}}, \Omega$ & $N$ & $A_{\mathrm{r}}, \mathrm{cm}^{2}$ & $l_{\mathrm{r}}, \mathrm{m}$ & $T_{\text {bias }}, \mathrm{MPa}$ \\
\hline 2 & 80 & 0 & 2.5 & 1840 & 1.27 & 0.144 & -70 \\
\hline
\end{tabular}

Table 2: Effective viscous damping factors for voltage-controlled stiffness switching.

\begin{tabular}{lc}
\hline System & Effective Viscous Damping Factor \\
\hline Controlled Response $1, H=75_{-55}^{+10} \mathrm{kA} / \mathrm{m}$ & 0.13 \\
Controlled Response $2, H=65_{-45}^{+10} \mathrm{kA} / \mathrm{m}$ & 0.10 \\
Controlled Response $2, H=65_{-45}^{+10} \mathrm{kA} / \mathrm{m}\left(F_{\text {mag }}\right.$ removed $)$ & 0.02 \\
\hline
\end{tabular}




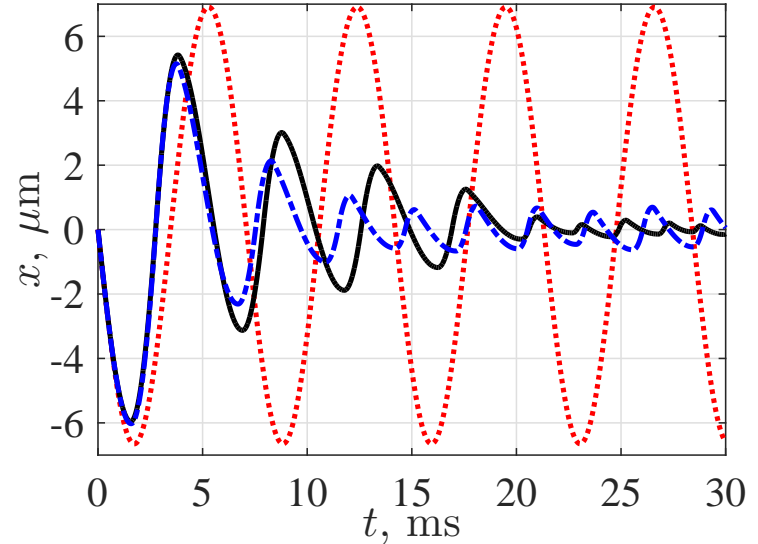

(a)

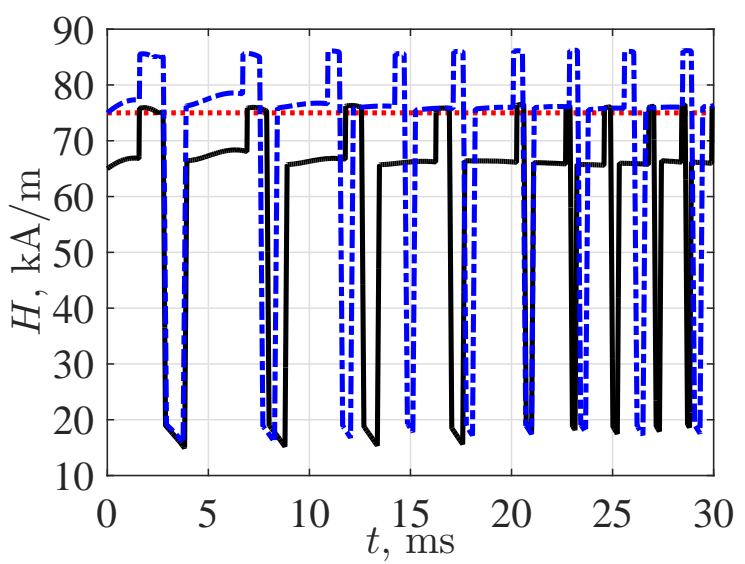

(c)

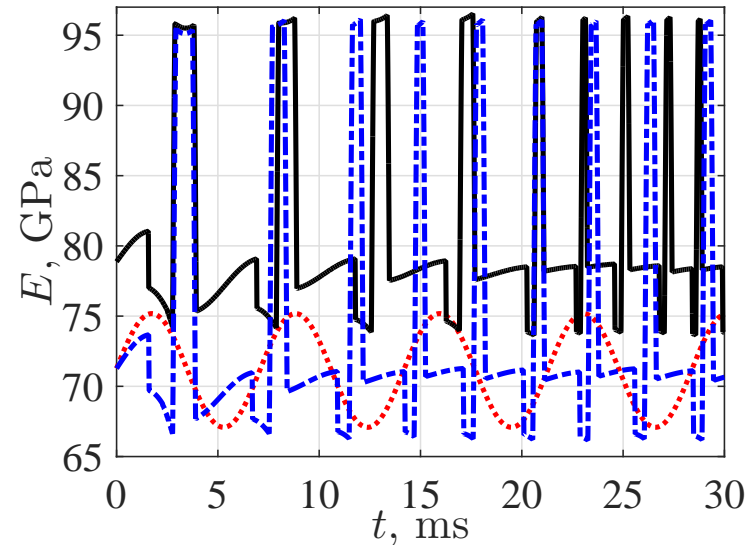

(b)

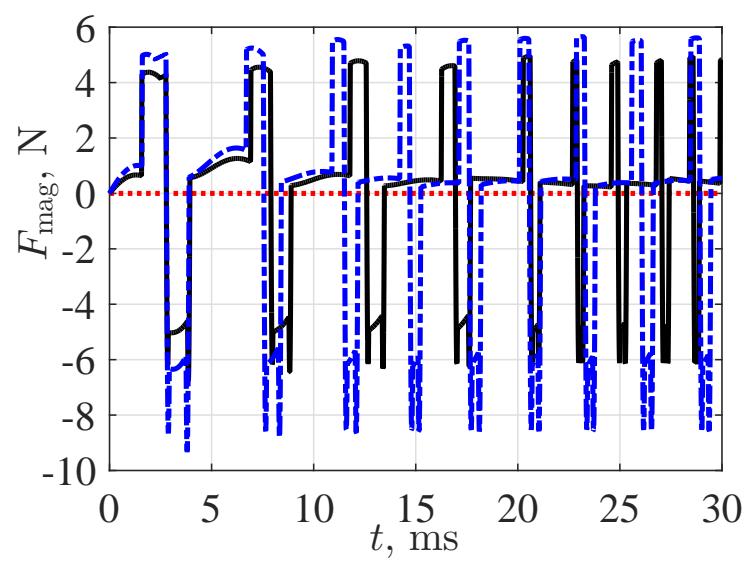

(d)

Figure 5: Enhanced decay of damped, free vibration using switched-stiffness vibration control: no control (dotted, $H_{\text {bias }}=75 \mathrm{kA} / \mathrm{m}$ ), controlled response 1 (dashed-dotted, $H=75_{-55}^{+10} \mathrm{kA} / \mathrm{m}$ ), and controlled response 2 (solid, $H=65_{-45}^{+10} \mathrm{kA} / \mathrm{m}$ ).

The effect of $F_{\mathrm{mag}}$ on the controlled response is further studied by solving the governing equations after artificially removing $F_{\text {mag }}$ from the mechanical equation (Eq. (12)). By doing so, the mechanical domain can influence the electrical domain, but the electrical domain only influences the mechanical domain through magnetic-field-induced changes in the stiffness $k^{H}$. Fig. 6 compares the uncontrolled response to controlled response 2 , with and without $F_{\text {mag. }}$. The effective viscous damping factors are reported in Table 2 . The magnetostrictive force significantly improves the control of free vibration by acting as an uncontrolled form of active vibration control.

As with the conventional control method, the performance of the modified control method improves with increases in the stiffness change magnitude. However, the magnetostrictive force can play a more important role than the stiffness change. In general, the performance of the modified control method improves as the amplitude of the magnetostrictive force is decreased by a change in the bias conditions or the range of the magnetic field. Optimizing the performance requires trial and error. 


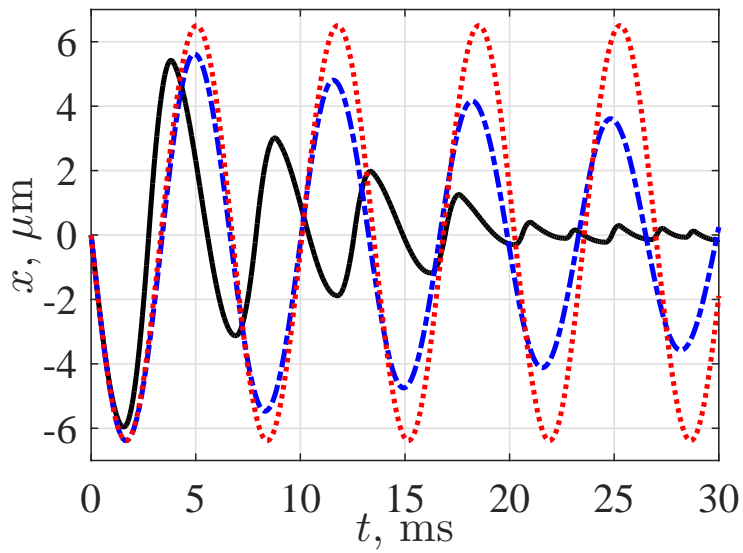

Figure 6: Effect of the magnetostrictive force on switched-stiffness vibration control of free vibration; no switching (dotted), and controlled response $2\left(H=65_{-45}^{+10} \mathrm{kA} / \mathrm{m}\right)$ with (solid) and without (dashed-dotted) the magnetostrictive force.

\subsection{Comparison with shunt-controlled stiffness switching and optimal resistive shunt damping}

Fukada et al. ${ }^{13}$ showed that the stiffness of magnetostrictive transducers can be controlled by shunting the electromagnet with a controllable inductor. In this case, the shunt operator is

$$
Z_{\mathrm{sh}}\left\{\Delta i_{\mathrm{sh}}\right\}=L_{\mathrm{sh}} \frac{d}{d t}\left(\Delta i_{\mathrm{sh}}\right)+\frac{d}{d t}\left(L_{\mathrm{sh}}\right) \Delta i_{\mathrm{sh}}
$$

When continuously tuning stiffness, the system that produces the shunt inductor (e.g., a circuit that controls the $V$-i relation using feedback) needs to capture the $d L_{\mathrm{sh}} / d t \cdot \Delta i_{\mathrm{sh}}$ term to allow for responsive tracking of the stiffness. However, when stiffness switching, this term acts as a very high bandwidth parametric excitation, which can work against the vibration control. To avoid the parametric excitation, stiffness can be changed by switching between open and short circuits (i.e., by switching between two resistive shunts). This is a key distinction between shunt-controlled and voltage-controlled stiffness switching, because an unwanted, high-frequency excitation cannot be avoided when switching with voltages (or currents). The open circuit stiffness $k^{H}$ and short circuit stiffness $k^{B}=k^{H}\left(1-\kappa^{2}\right)$ bound the range of stiffness that is possible using a shunt with positive inductance. ${ }^{\S}$ Thus, the energy loss due to stiffness switching is maximized by switching between open and short circuits.

Fig. 7 presents a comparison of voltage-controlled stiffness switching, shunt-controlled stiffness switching, and optimal resistive shunt damping. The conventional switched-stiffness control law is used for shunt switching, because it does not introduce a magnetostrictive force. Two forms of shunt control are considered: switching between open and short circuits and switching between open circuit and optimal resistive shunt. ${ }^{7}$ Here, the magnetomechanical bias was moved to the center of the burst region where the coupling factor $\kappa$ is maximized, because shunt damping and shunt-controlled stiffness changes improve with increases in $\kappa$. As a result, the nominal stiffness of the shunt-controlled transducer is considerably smaller than that of the voltage-controlled transducer. The free vibration response is shown in Fig. 7a. Optimal resistive shunt damping clearly performs the best, but voltage-controlled switching performs considerably better than either form of shunt-controlled switching. Switching between open and short circuits attenuates the free vibration slightly faster than switching between open and optimal resistive shunts, although energy is dissipated in the former case only during stiffness switches. Effective viscous damping factors are shown in Table 3 for completeness. However, it is more insightful to investigate the kinetic energy of the mass $\left(0.5 m \frac{d}{d t}(\Delta x)^{2}\right)$,

\footnotetext{
${ }^{\S}$ Larger stiffness changes are possible if a negative inductance shunt is used, but these shunts are not considered in this paper.
} 


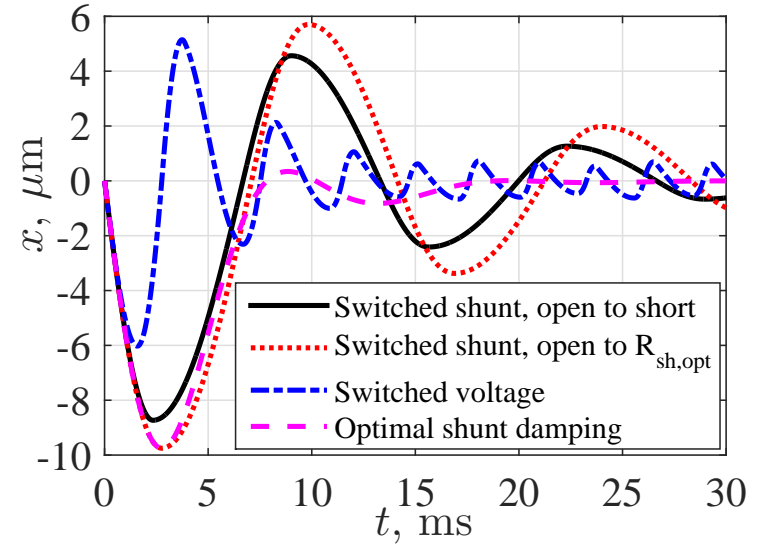

(a)

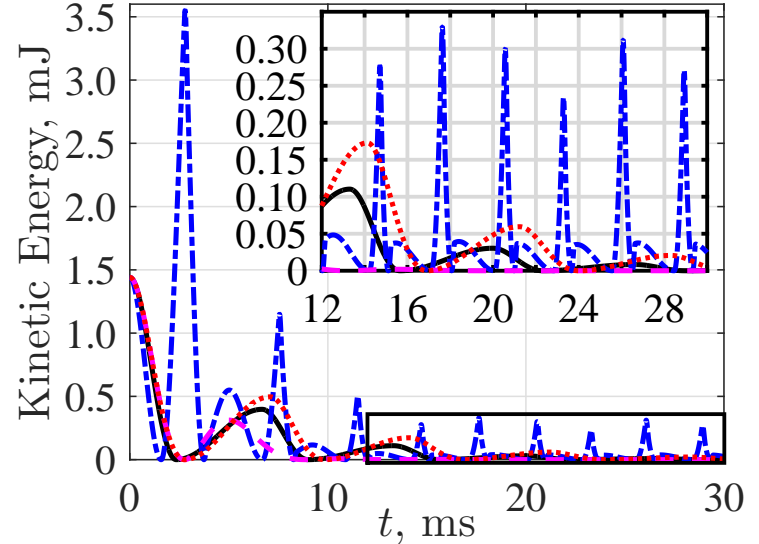

(b)

Figure 7: Comparison of voltage-controlled stiffness switching (controlled response 1) to shunt-controlled stiffness switching and optimal resistive shunt damping; $T_{\text {bias }}=-25 \mathrm{MPa}, H_{\text {bias }}=40 \mathrm{kA} / \mathrm{m}, \kappa_{\text {bias }}^{2}=0.751$.

because there is a large difference in the oscillation frequencies. Fig. 7b shows how each control technique dissipates the kinetic energy of the mass. If the voltage-controlled stiffness switching was turned off and the electromagnet was shorted at about $12 \mathrm{~ms}$, the oscillation energy would be sufficiently dissipated by about 14 to $16 \mathrm{~ms}$. However, the shunt-controlled switching techniques do not sufficiently dissipate the oscillation energy until about 22 to $24 \mathrm{~ms}$.

Table 3: Effective viscous damping factors for voltage- and shunt-controlled stiffness switching.

\begin{tabular}{lc}
\hline System & Effective Viscous Damping Factor \\
\hline Switched shunt, open to short & 0.20 \\
Switched shunt, open to $R_{\text {sh,opt }}$ & 0.17 \\
Switched voltage (controlled response 1) & 0.13 \\
Optimal shunt damping & 0.37 \\
\hline
\end{tabular}

\section{SUMMARY AND CONCLUSIONS}

A computational study of switched-stiffness vibration control using a magnetostrictive transducer was conducted. Two methods of stiffness switching were considered: changing the bias magnetic field using a voltage excitation and changing the impedance of an electrical shunt connected to the transducer's electromagnet. The transducer was implemented as a tunable spring mount to dampen the vibration of a single-degree-offreedom mechanical system. A model was presented that incorporates constitutive nonlinearities, mechanical vibrations, the electrical dynamics associated with voltage control or shunt control of the transducer, and the magnetostrictive force generated by the transducer. To greatly simplify the model and numerical method, mechanically-induced and field-induced eddy current effects were neglected. According to the analytical and experimental findings of Scheidler et al. ${ }^{9}$ this is a valid assumption for Terfenol-D-based transducers operated at forcing frequencies up to about $1 \mathrm{kHz}$.

Voltage-controlled stiffness switching was studied in detail. Free vibration of the mass under passive damping and switched-stiffness vibration control was calculated. The magnetostrictive force, which is unwanted, but accompanies field-induced stiffness changes, was shown to play a significant role in the performance of the control. If the net magnetostrictive force over one vibration cycle is not small, the force can resonate the mass by overcoming the energy lost due to passive damping and stiffness switching. Thus, a 
modified control law was proposed to minimize the influence of the magnetostrictive force. Stiffness switching according to the modified law was found to introduce a level of damping that is similar to an effective viscous damping factor of up to 0.13 . Performance may be improved if the bias magnetic field is adjusted by exciting the transducer with a controlled current source rather than a controlled voltage source. In general, the performance of the control improved as the stiffness change magnitude increased and the amplitude of the magnetostrictive force decreased. Although, the magnetostrictive force can significantly improve vibration attenuation by acting as a very simple form of active vibration control; from this perspective, the magnetostrictive force is a partially-controlled external force generated by external energy. For the magnetostrictive force to improve attenuation, the modified control law must be used and the bias conditions and magnetic field changes must be carefully selected. In practice, it may be difficult to achieve good performance due to time delays in the feedback loop and to uncertainty and noise in the determination of the mechanical states; it may be particularly difficult when the structure to be controlled vibrates at multiple modes.

The effectiveness of voltage-controlled stiffness switching was compared to that of shunt-controlled stiffness switching and optimal resistive shunt damping. The results show that voltage control performs better than shunt control due to the magnetostrictive force. Also, large changes in modulus can be achieved using voltage or current control. However, voltage control and current control require external energy. Shunt control can only provide moderate modulus changes and it generates a parametric force if reactive shunts are used. Nevertheless, shunt control can be easily realized with synthetic impedance shunts, which use a feedback circuit to actively control the current-voltage relation of the shunt; these circuits require very little energy, which can be harvested from the mechanical vibration using the transducer. Moreover, shunt control does not introduce a magnetostrictive force (i.e., an external force) and does not produce a parametric force if resistive shunts are used. Thus, compared to voltage control and current control, shunt control should be easier to realize in practice and more robust to error in mechanical state sensing, to more complex loading, and to changes in operating conditions. Both stiffness tuning methods can also provide continuous stiffness tuning in addition to stiffness switching. However, one benefit of voltage-controlled (or current-controlled) switching over voltage-controlled continuous tuning is that a switching amplifier can be used rather than a linear amplifier, which are heavier, bulkier, and more expensive.

For the cases considered in this paper, optimal resistive shunt damping performed considerably better than both voltage- and shunt-controlled stiffness switching. Shunt damping is also a fully passive technique that is compact and very simple to implement. This suggests that there may not be a benefit to using stiffness switching techniques. Accordingly, future work should investigate the performance of these techniques under forced vibration over a broad frequency range to determine if this trend applies generally. Alternatively, future work can study whether the best vibration control performance is achieved by concurrently implementing optimal shunt damping and voltage-controlled (or current-controlled) stiffness switching.

The constitutive model used in this study is anhysteretic. Energy loss inside the magnetostrictive material - namely eddy currents, magnetic hysteresis, and mechanical material damping - will reduce the magnetomechanical energy conversion, which should negatively affect the peformance of shunt damping and shunt-controlled stiffness switching. However, it is unclear how the presence of these internal loss mechanisms affects the total energy dissipated and therefore the overall vibration attenuation. This is a possible topic of future work.

\section{ACKNOWLEDGMENTS}

This work was supported by NASA contract \# NNC13BA10 and the NASA Aeronautics Scholarship Program (grant \# NNX14AE24H). Additional support was provided by the NASA Aeronautics Research Mission Directorate's Revolutionary Vertical Lift Technology project and the Smart Vehicle Concepts Center (www.SmartVehicleCenter.org), a National Science Foundation Industry/University Cooperative Research Center. 


\section{REFERENCES}

[1] Scheidler, J. and Dapino, M., "Nonlinear dynamic modeling and resonance tuning of Galfenol vibration absorbers," Smart Materials and Structures 22(8), 085015 (2013).

[2] Kellogg, R. and Flatau, A., "Wide band tunable mechanical resonator employing the $\delta$ E effect of Terfenol-D," Journal of Intelligent Material Systems and Structures 15(5), 355-368 (2004).

[3] Flatau, A. B., Dapino, M. J., and Calkins, F. T., "High bandwidth tunability in a smart vibration absorber," Journal of Intelligent Material Systems and Structures 11(12), 923-929 (2000).

[4] Pagliarulo, P., Kuhnen, K., May, C., and Janocha, H., "Tunable magnetostrictive dynamic vibration absorber," in [Proceedings of the 9th International Conference on New Actuators], 367-370 (2004).

[5] Wright, R. and Kidner, M., "Vibration absorbers: a review of applications in interior noise control of propeller aircraft," Journal of Vibration and Control 10(8), 1221-1237 (2004).

[6] Scheidler, J. J. and Dapino, M. J., "Stiffness tuning of FeGa structures manufactured by ultrasonic additive manufacturing," in [Proceedings of SPIE], 9059, 905907 (2014).

[7] Clark, W. W., "Vibration control with state-switched piezoelectric materials," Journal of Intelligent Material Systems and Structures 11(4), 263-271 (2000).

[8] Ramaratnam, A. and Jalili, N., "A switched stiffness approach for structural vibration control: theory and real-time implementation," Journal of Sound and Vibration 291(1), 258-274 (2006).

[9] Scheidler, J. J., Asnani, V. M., and Dapino, M. J., "Dynamically tuned magnetostrictive spring with electrically controlled stiffness," Smart Materials and Structures 25(3), 035007 (2016).

[10] Chakrabarti, S. and Dapino, M., "Fully coupled discrete energy-averaged model for Terfenol-D," Journal of Applied Physics 111(5), 054505-054505 (2012).

[11] Evans, P. and Dapino, M., "Efficient magnetic hysteresis model for field and stress application in magnetostrictive Galfenol," Journal of Applied Physics 107(6), 063906 (2010).

[12] Tari, H., Scheidler, J., and Dapino, M., "Robust solution procedure for the discrete energy-averaged model on the calculation of 3D hysteretic magnetization and magnetostriction of iron-gallium alloys," Journal of Magnetism and Magnetic Materials 384, 266-275 (2015).

[13] Fukada, E., Date, M., and Sekigawa, K., "Vibration control by magnetostrictive actuator coupled with negative inductance circuits," Japanese Journal of Applied Physics 42(11R), 7124 (2003).

[14] Hagood, N. W. and von Flotow, A., "Damping of structural vibrations with piezoelectric materials and passive electrical networks," Journal of Sound and Vibration 146(2), 243-268 (1991).

[15] Scheidler, J. J. and Dapino, M. J., "Mechanically induced magnetic diffusion in cylindrical magnetoelastic materials," Journal of Magnetism and Magnetic Materials 397, 233-239 (2016).

[16] Scheidler, J. J., Static and Dynamic Delta E Effect in Magnetostrictive Materials with Application to ElectricallyTunable Vibration Control Devices, PhD thesis, The Ohio State University (2015).

[17] Engdahl, G., ed., [Handbook of Giant Magnetostrictive Materials], Academic Press, San Diego, CA (2000). 